\title{
AVALIAÇÃO NUTRICIONAL NO PACIENTE CIRRÓTICO: uma abordagem objetiva, subjetiva ou multicompartimental?
}

\author{
Luciana RITTER e Jussara GAZZOLA
}

RESUMO - Racional - A desnutrição decorrente da doença hepática é freqüente, porém sua detecção é difícil. Os parâmetros de avaliação nutricional comumente utilizados na prática clínica têm seu uso limitado em pacientes cirróticos. Várias das manifestações clínicas da desnutrição são resultantes da doença hepática, o que tende a confundir e alterar o diagnóstico nutricional. Objetivos - Apresentar uma revisão dos diferentes métodos de avaliação nutricional, bem como suas limitações e/ou aplicações em pacientes cirróticos. Conclusão - Dos métodos apresentados, o modelo de avaliação multicompartimental de quatro compartimentos se mostrou bastante eficiente na detecção de desnutrição. Entretanto, apesar dos bons resultados apresentados, os métodos usados para sua detecção ainda esbarram em dificuldades técnicas e de alto custo. A força de aperto de mão não-dominante (dinamometria) aparece como método alternativo pelo baixo custo, simplicidade e eficiência na detecção de desnutridos ou pacientes em risco de desnutrição. É o método mais sensível por predizer uma incidência significativa de complicações em pacientes cirróticos desnutridos.

DESCRITORES - Avaliação nutricional. Cirrose hepática. Força da mão.

\section{INTRODUÇÃO}

A avaliação nutricional é a peça chave do profissional nutricionista para o manuseio do seu paciente, pois é através dela que se determina o diagnóstico nutricional no qual irá se basear a conduta de correção nutricional adequada. A avaliação nutricional objetiva é baseada em parâmetros comumente utilizados, tais como: peso, altura, relação peso-altura (índice de massa corporal - IMC), medidas de pregas cutâneas, da circunferência do braço, circunferência muscular do braço e o índice creatinina/altura. Incluem-se também os dados da anamnese nutricional do paciente e o exame físico, que investiga sinais de deficiências nutricionais. De acordo com JEEJEEBHOY et al. ${ }^{(18)}$, apenas um dado antropométrico é insuficiente para classificar um indivíduo como desnutrido. São necessários pelo menos três para que se possa concluir por desnutrição. O IMC, no entanto, sozinho é considerado como bom parâmetro para a avaliação nutricional ${ }^{(1)}$. Aliados a esses dados, alguns exames laboratoriais são também utilizados: proteínas plasmáticas (albumina, pré-albumina, transferrina, hemoglobina e hematócrito), métodos imunológicos (contagem total de linfócitos, testes de hipersensibilidade cutânea tardia) e balanço nitrogenado.

Freqüentemente, devido às condições do paciente, $o$ profissional tem dificuldades de obter dados objetivos para definir seu estado nutricional (pesar, medir, obter outros dados antropométricos ou exames laboratoriais). Aplica, então, a avaliação nutricional subjetiva global (ANSG), uma forma simples de se avaliar aspectos nutricionais e se chegar ao diagnóstico nutricional.

A avaliação nutricional multicompartimental ocorre através da observação de variações nos compartimentos corporais (massa celular corporal (MCC), água corporal total, gordura corporal, conteúdo mineral ósseo).

A avaliação nutricional de pacientes com cirrose hepática é considerada difícil ${ }^{(22)}$. Não existe, até o momento, um padrão-ouro para a estimativa do seu estado de nutrição ${ }^{(1)}$. A desnutrição decorrente da doença hepática é freqüente. ROONGPISUNTHIPONG et al. ${ }^{(27)}$ sugerem que a prevalência da desnutrição protéico-calórica (DPC) em doenças hepáticas crônicas situe-se entre 10\% a $100 \%$, figurando como fator de alto risco para eles. A

Endereço para correspondência: Dra. Luciana Ritter - Rua Padre Paulo Englert. 35 - Bairro Ipanema - 91770-770 - Porto Alegre, RS. E-mail: luciana_ritter@yahoo.com.br 
desnutrição nesses pacientes é comum, mas freqüentemente não diagnosticada ${ }^{(22)}$. Está associada a altas taxas de morbidade e mortalidade relacionadas com o transplante hepático e à cirurgia abdominal, influenciando na sobrevida a curto e longo prazos. Especificamente em pacientes cirróticos a prevalência de desnutrição é alta, independente da causa ${ }^{(22,27)}$. O diagnóstico da desnutrição nesse grupo e a intervenção terapêutica precoce são essenciais para a correção nutricional ${ }^{(2)}$.

Os parâmetros de avaliação nutricional normalmente empregados na prática clínica (IMC, perimetria, exames laboratoriais) têm seu uso limitado em pacientes cirróticos. Várias das manifestações clínicas da desnutrição são decorrentes, tão-somente, da doença hepática o que tende a confundir e alterar seu diagnóstico nutricional. Embora poucos estudos tenham sido feitos sobre a avaliação da composição corporal na doença hepática crônica, espera-se que a análise da composição corporal possa proporcionar informações adicionais e mais precisas sobre o estado nutricional destes pacientes ${ }^{(12)}$.

Para BORGES et al. ${ }^{(6)}$ não existe consenso geral sobre qual método de avaliação nutricional estaria indicado. Segundo PAROLIN et al. ${ }^{(26)}$ o nutricionista é o profissional capacitado para utilizar o maior número possível de parâmetros para avaliar esses pacientes, a fim de aumentar a confiabilidade dos resultados.

\section{Cirrose e desnutrição}

O fígado assume posição anatômica estratégica na circulação portal e sistêmica. É o centro de regulação dos nutrientes ingeridos, órgão essencialmente metabólico. Através da circulação porta, o fígado recebe vários nutrientes e, após a absorção, retém alguns para reserva e metabolismo próprios e distribui outros para circulação sistêmica, para eventual utilização ou depósitos em outros tecidos, exercendo papel central na metabolização dos nutrientes. Uma vez que ocorra dano funcional no fígado, esse mecanismo será afetado, podendo levar a desnutrição.

Na cirrose hepática, que se caracteriza por alteração crônica e irreversível do parênquima hepático, todo este mecanismo se altera pois tanto a estrutura hepática, quanto o funcionamento dos hepatócitos e a circulação porta se modificam. O risco de desnutrição em cirróticos é grande.

A causa da cirrose hepática pode ser alcoólica, criptogênica e pós-hepatite, biliar, cardíaca, metabólica, hereditária e relacionada $\operatorname{com}_{\text {fármacos }}{ }^{(9)}$. No estudo conduzido por BRANDÃO et al ${ }^{(8)}$, em amostra de 113 pacientes cirróticos adultos, 29,2\% apresentavam cirrose devido ao uso de álcool concomitante com a infecção viral (por vírus da hepatite $\mathrm{B}$ e $\mathrm{C}$ ). $\mathrm{O}$ vírus da hepatite $\mathrm{C}$ foi encontrado em $24,8 \%$ dos pacientes, uma vez que somente o álcool foi causa de $22,1 \%$ dos casos. A cirrose por infecção por vírus $\mathrm{B}$ foi encontrada apenas em $4,42 \%$ dos pacientes.

A DPC ocorre quando há ingestão inadequada de calorias, proteínas ou ambas, de acordo com as necessidades nutricionais do indivíduo. A DPC pode levar a dano hepático uma vez que o indivíduo se torna mais suscetível a infecções e agentes tóxicos. $\mathrm{McCULLOUGH}^{(22)}$ cita que a DPC levaria à deterioração mais rápida da função hepática. Ou seja, há a formação de um ciclo vicioso, onde a desnutrição agrava a doença e essa agrava o estado nutricional.
A presença de ascite com seu efeito compressivo contribui para a saciedade precoce, assim como a encefalopatia e hemorragias digestivas facilitariam uma inadequada ingestão de nutrientes. As dietas administradas visando o tratamento das complicações, como a dieta pobre em proteínas para a encefalopatia, a pobre em sódio e fluidos para ascite e edema, também contribuiriam para a instalação da DPC. Em estudo conduzido por McCULLOUGH ${ }^{(22)}$, também se incluiriam outros fatores comumente encontrados em hepatopatas, como: anorexia $(87 \%)$, perda de peso $(60 \%)$ e náusea $(55 \%)$. A desnutrição pode também advir de um estado de hipermetabolismo, condição encontrada na minoria de pacientes cirróticos - $16 \%$ a $34 \%$ de pacientes.

A ingestão inadequada de zinco, diminuindo a palatabilidade também contribui para a $\mathrm{DPC}^{(6)}$. Como demonstrado por GOTTSCHALL et al. ${ }^{(16)}$, em amostra de 34 pacientes cirróticos, todos apresentavam ingestão de zinco menor que $35 \%$ da Recommended Dietary Allowances.

Na Tabela 1 são apresentados alguns dados compilados sobre a prevalência de desnutrição em hepatopatas.

TABELA 1 - Prevalência de desnutrição em hepatopatias

\begin{tabular}{lccc}
\hline Autor (ano) & Amostra & Critério & Prevalência \\
\hline Morgan et al.(1976) & 80 & Antrop e bioq & $40 \%-60 \%$ \\
Mendenhall et al.(1984) & 363 & Antrop e bioq & $86 \%-100 \%$ \\
IMCPNC (1994) & 1402 & AMB ou AGB & $30 \%$ \\
Merli et al.(1996) & 1492 & AMB e/ou AGB & $35 \%$ \\
Tresierra et al.(2002) & 43 & Antrop e bioq & $65 \%$ \\
Leitão et al.(2003) & 32 & CB, CMB, PCT & $63 \%$ \\
\hline
\end{tabular}

Antrop. $=$ parâmetros antropométricos; Bioq. = parâmetros bioquímicos;

$\mathrm{PCT}=$ prega cutânea tricipital; $\mathrm{CMB}=$ circunferência muscular do braço; $A M B=$ área muscular do braço; $A G B=$ área de gordura do braço; $\mathrm{CB}=$ circunferência do braço

\section{Parâmetros utilizados na avaliação nutricional em pacientes cirróticos}

\section{Avaliação objetiva}

A retenção hídrica, edema e ascite, comumente observados nos estágios finais da doença, podem também aparecer nos estágios iniciais. Figuram como o grande fator de confusão que cancela o uso dos parâmetros de percentagem de peso ideal e IMC, por subestimar a gravidade da desnutrição e sua prevalência. Para VENEGAS-TRESIERRA et al. ${ }^{(28)}$, em amostra de 43 pacientes cirróticos hospitalizados, foram encontrados $30,2 \%$ de obesos, $20,1 \%$ com sobrepeso, $46,5 \%$ de eutróficos e apenas $3,2 \%$ com baixo peso. $O$ estudo não excluía os pacientes cirróticos com ascite. Para ROONGPISUTHIPONG et al. ${ }^{(27)}$, a prevalência de DPC em pacientes com cirrose hepática ficou em torno de 13,3\%, segundo o parâmetro percentagem de peso ideal $(<90 \%)$ e $11,7 \%$ pelo parâmetro IMC $\left(<18,5 \mathrm{~kg} / \mathrm{m}^{2}\right)$, subestimando o número de desnutridos da amostra.

Como mostrado por GOTTSCHALL et al. ${ }^{(16)}$, numa série de 34 indivíduos com cirrose pelo vírus da hepatite $C, 4$ destes apresentando ascite, não foi encontrado nenhum desnutrido utilizando-se o parâmetro IMC. Pelo contrário, foi encontrada alta prevalência de sobrepeso $(62 \%)$. 
A retenção hídrica, por sua vez, não influencia as medidas da prega cutânea triciptal (PCT), prega cutânea subescapular, circunferência muscular do braço (CMB) e circunferência do braço (CB), quando não existe edema generalizado. Segundo BRAMLEY et al.$^{(7)}$, independente da retenção hídrica vigente, foi encontrada alta correlação entre tecido adiposo corporal estimada por raios-X e a obtida pela PCT em pacientes cirróticos, com ou sem ascite. Para MERLI et al. ${ }^{(24)}$, a CMB seria bom indicador de desnutrição grave em pacientes nos estágios iniciais da doença, visto que a depleção de tecido muscular mostrou correlação com altas taxas de mortalidade nesses pacientes. De fato, o uso desses parâmetros não seria fidedigno em estágios finais da cirrose, pois nesses pacientes ocorre utilização aumentada de lipídios endógenos, podendo alterar tais parâmetros ${ }^{(25)}$. Ademais, há grande variação entre as medidas para avaliação do estado nutricional feitas por diferentes observadores e faltam equações específicas para pacientes com doença hepática, uma vez que os estudos para validação destas equações utilizaram pessoas saudáveis ${ }^{(15)}$.

$\mathrm{O}$ índice creatinina/altura, parâmetro adequado para a estimativa da massa muscular ou celular corpórea, deve ser excluído da avaliação nutricional, no caso de déficit de função renal, complicação geralmente observada nestes pacientes e que limita a aplicação deste método.

As proteínas viscerais são claramente influenciadas pela capacidade de síntese hepática, prejudicando seu uso na avaliação nutricional. Seu uso reflete a disfunção hepática, devido ao dano hepático, e não necessariamente o estado nutricional. A albumina, considerada parâmetro fiel na avaliação nutricional ${ }^{(10)}$, sintetizada no fígado, tem sua síntese diminuída, seu catabolismo acelerado, diminuindo a confiabilidade do teste para avaliação nutricional em pacientes cirróticos. É utilizada por diversos autores ${ }^{(23,28)}$ como marcador de desnutrição, mesmo em hepatopatas, onde $3,5 \mathrm{~g} / \mathrm{dL}$ é o parâmetro para desnutrição utilizado.

Hematócrito e hemoglobina aparecem como bons indicadores nutricionais mesmo para hepatopatas, pois independem do metabolismo hepático. Estudo conduzido na Universidade Estadual Paulista ${ }^{(20)}$ mostrou que esses parâmetros, assim como a CMB e a prega cutânea subescapular, seriam os ideais para a avaliação do estado nutricional em pacientes cirróticos. No entanto, a hemoglobina e o hematócrito poderiam estar alterados em caso de hipertensão portal grave acompanhada de esplenomegalia maciça e hiperesplenismo, bem como na presença de hemorragias digestivas.

Os testes de imunidade, como a contagem absoluta de linfócitos, assim como os testes cutâneos de hipersensibilidade tardia (cândida, PPD), refletem a higidez do estado nutricional. Como descrito por PAROLIN et al. ${ }^{(26)}$, a presença de infecções, desequilíbrio hidroeletrolítico, insuficiência renal, estresse metabólico, doenças inflamatórias intestinais e imunossupressão, condições normalmente observadas em hepatopatias, podem alterar os resultados, comprometendo a avaliação nutricional. A imunidade celular e a contagem total de linfócitos são também influenciadas pela doença hepática e pelo alcoolismo.

As primeiras alterações nutricionais ocorrem dentro da célula muscular, com reflexo em sua função. Dessa forma, a medição da capacidade muscular é bom método de avaliação nutricional e potencialmente independente da doença hepática ${ }^{(2)}$.

Inicialmente, o uso do dinamômetro ou força de aperto de mão não-dominante (FAM), como ferramenta para avaliação nutricional, poderia sofrer a interferência de fatores que não a desnutrição. A disfunção muscular refletiria a presença de uma neuropatia periférica e a própria presença da doença hepática, com conseqüentes alterações metabólicas, poderia anular seu uso como indicador de estado nutricional. Para ANDERSEN et al. ${ }^{(4)}$, a neuropatia periférica, assim como a gravidade da doença hepática não influenciaria na perda de força muscular em cirróticos, sendo esta relacionada à severidade da desnutrição. $\mathrm{O}$ uso da dinamometria, por sua vez, seria bom método. Segundo ÁLVARES-da-SILVA ${ }^{(1)}$, o uso da FAM é método simples, barato e eficaz para a detecção de desnutrição em pacientes cirróticos ambulatoriais. Em seu estudo, mostrou-se mais sensível no diagnóstico de desnutrição em detrimento de outros métodos, dados confirmados por GOTTSCHALL et al. ${ }^{(16)}$. Segundo estes, o diagnóstico de desnutrição medido pela FAM foi superior $(79,4 \%)$ aos demais métodos utilizados (ANSG, PCT, CMB, IMC), refletindo a capacidade desse método de diagnosticar desnutrição na ausência de qualquer evidência clínica ${ }^{(16)}$.

ÁLVARES-da-SILVA e SILVEIRA ${ }^{(3)}$ encontraram $63 \%$ de desnutridos com o uso da FAM, que poderiam ser explicados pela baixa especificidade da dinamometria (45\%), quando comparada à $\mathrm{ANSG}^{(3)}$, ou seja, pode-se erroneamente diagnosticar como desnutridos pacientes que não o são. Para esses mesmos autores, após 1 ano, foi encontrada incidência significativa maior de complicações da doença hepática em pacientes considerados desnutridos pelo uso da FAM, demonstrando a efetividade desse método em identificar pacientes mais susceptíveis ao desenvolvimento de complicações.

\section{Avaliação subjetiva}

A ANSG, proposta por DETSKY et al. ${ }^{(11)}$, é método prático de se obter diagnóstico nutricional do paciente, quando o acesso aos dados objetivos de avaliação nutricional está restrito. Sua aplicabilidade em pacientes cirróticos e nos candidatos a transplante hepático seria válida, uma vez que a doença hepática altera a maioria dos dados objetivos normalmente empregados.

A ANSG consiste na prática da anamnese e do exame físico, em que se avaliam, principalmente, perda de peso, tecido adiposo, muscular e alterações na ingestão dietética. Deve ser realizada por observador treinado, sendo procedimento de baixo custo. Segundo COPPINI et al. ${ }^{(10)}$, a ANSG apresentou associação significativa com a hipoalbuminemia $(P<0,05)$, o que passou a gerar maior confiança neste método na detecção acurada de desnutrição. BARBOSA-SILVA e BARROS ${ }^{(5)}$ estabeleceram a ANSG como boa opção para avaliação nutricional em hepatopatas. As informações da história do paciente poderiam partir de seus familiares, no caso de presença de encefalopatia. Porém, a presença de ascite e retenção hídrica dificultam a aplicabilidade da avaliação subjetiva. A estimativa de perda de peso, que faz parte desta avaliação fica difícil de ser estimada nestes pacientes ${ }^{(16)}$.

A Tabela 2 mostra os fatores que influenciam a avaliação nutricional em indivíduos com cirrose hepática. 
TABELA 2 - Avaliação nutricional em cirrose

\begin{tabular}{lcccc}
\hline Medidas & $\begin{array}{c}\text { Desnutrição } \\
\text { protéica }\end{array}$ & DPC & Alcoolismo & Hepatopatia \\
\hline Proteínas viscerais & $\mathrm{X}$ & & $\mathrm{X}$ & $\mathrm{X}$ \\
CTL & $\mathrm{X}$ & & $\mathrm{X}$ & $\mathrm{X}$ \\
Imunid. celular & $\mathrm{X}$ & & $\mathrm{X}$ & $\mathrm{X}$ \\
$\%$ peso ideal & & $\mathrm{X}$ & & $\mathrm{X}$ \\
Antropometria & & $\mathrm{X}$ & & $?$ \\
Índice creatinina-altura & & $\mathrm{X}$ & & $?$ \\
ANSG & & $\mathrm{X}$ & & $\mathrm{X}$ \\
BIA & & $\mathrm{X}$ & & $\mathrm{X}$ \\
\hline
\end{tabular}

McCullough (2000)|221

$X=$ fatores que afetam a eficácia das medidas $\quad ?=$ sugerido

$\mathrm{CLT}=$ Contagem total de linfócitos

ANSG = Avaliação nutricional subjetiva global

$\mathrm{BIA}=$ Bioimpedância elétrica

\section{Avaliação multicompartimental}

A avaliação multicompartimental baseia-se na observação das alterações presentes nos compartimentos corporais.

A maioria dos estudos de DPC envolve técnicas baseadas no modelo de dois compartimentos quimicamente distintos, no qual o peso corporal é dividido em massa de gordura e massa sem gordura. A primeira pode ser avaliada pela antropometria, através da mensuração das pregas cutâneas, e a segunda pelo índice de creatinina, pelas circunferências musculares (proteína somática), e pela dosagem de proteínas séricas (proteína visceral). A grande limitação do modelo de dois compartimentos é que ele pressupõe que os componentes dentro da massa sem gordura estão presentes na mesma proporção em todos os indivíduos. Porém, inúmeras condições, tais como idade, gravidez, obesidade levam a erros nestas estimativas, fazendo sub ou superavaliações dos componentes e tornando esse modelo impreciso na avaliação da composição corporal ${ }^{(17)}$.

Atualmente, os autores propõem avaliações baseadas em modelos de quatro a seis compartimentos. Para FIGUEIREDO ${ }^{(12)}$, o uso do modelo de quatro compartimentos (MCC, água corporal total - intracelular e extracelular, gordura corporal e conteúdo mineral ósseo), apresenta a grande vantagem do baixo custo, quando comparado ao modelo de seis compartimentos que utiliza a análise de ativação neutrônica.

A cirrose hepática é caracterizada por alteração nos principais compartimentos corporais, evidenciando-se aumento na água corporal total (especialmente extracelular) e diminuição na MCC e gordura corporal total. Outra alteração importante na cirrose é a perda da massa óssea. Infelizmente nenhum parâmetro de avaliação nutricional tradicional é capaz de prever essas alterações ${ }^{(13)}$.

A MCC é o compartimento metabolicamente ativo, composto principalmente por músculo. Ela reflete os componentes celulares responsáveis pela transferência de energia e trabalho bioquímico, sendo considerada ótima referência para expressar as taxas dos processos fisiológicos como o gasto de energia e a proteólise ${ }^{(21)}$. Como a gordura corporal funciona como reserva de energia, as medidas da MCC e da gordura corporal, figuram como excelente indicador de estado nutricional, uma vez que a depleção dos mesmos indica desnutrição.

A bioimpedância elétrica (BIA) é método seguro, barato, não-invasivo e rápido para a determinação do compartimento de água corporal total. Em pacientes cirróticos com ascite ou retenção hídrica, seu uso é limitado, já que, por apresentarem alterações na distribuição da água intracelular e extracelular, os valores da BIA não são confiáveis ${ }^{(19)}$.

Em estudo conduzido por FIGUEIREDO ${ }^{(12)}, 69$ pacientes tiveram sua MCC medida através da técnica de diluição isotópica, para estimar a água intracelular, bem como a gordura corporal. Estes resultados foram comparados com as técnicas de avaliação nutricional tradicionais (ANSG, PCT, CB, CMB, teste laboratoriais, absortímetro de dupla energia de raios- $\mathrm{X}$ e dinamometria). Foi encontrada pouca relação entre as medidas de $\mathrm{MCC}$ e as encontradas pelos métodos tradicionais. Apenas o dinamômetro e a CMB se mostraram sensíveis na detecção de perdas de MCC nesses pacientes.

O DEXA (absortímetro de dupla energia de raios- $\mathrm{X}$ ), usado para medir a gordura corporal e o conteúdo mineral do osso, através da emissão de raios-X em duas freqüências diferentes, é também bom método de avaliação. Sua limitação está, como na BIA, na não distinção entre água intracelular e extracelular, bem como no alto custo dos equipamentos, falta de disponibilidade e exposição à radiação que seu uso proporciona ${ }^{(13)}$. Em contraponto, BORGES et al. ${ }^{\left({ }^{6}\right.}$ citam que a exposição à radiação é mínima ( $<1 \mathrm{mrem})$, permitindo que seja empregado em estudos seriados e com crianças.

A técnica do potássio corporal total utiliza o pressuposto que o potássio está presente na massa magra $(54-60 \mathrm{mmol} / \mathrm{kg}$ na mulher e $64-68 \mathrm{mmol} / \mathrm{kg}$ no homem) e ausente no tecido adiposo. Entretanto, sua concentração é maior nos músculos do que nos outros tecidos e, além disso, há dúvidas se o potássio corporal total seria medida confiável em pacientes com cirrose hepática devido à ocorrência freqüente de hipopotassemia nestes pacientes, o que resultaria em subestimação da massa magra. Além disso, é técnica cara, de grande dificuldade operacional, com chance de erro nas medidas ${ }^{(12)}$.

Em outro estudo conduzido por FIGUEIREDO et al. ${ }^{(14)}$, o uso da avaliação nutricional dos compartimentos foi sensível o bastante na detecção de desnutrição, mesmo em pacientes em estágios iniciais de doença hepática, bem como naqueles com desnutrição não detectada clinicamente por outros métodos. Foram observadas perda significativa de gordura nos estágios iniciais da doença (Child A) e acelerada perda de massa celular nos estágios mais avançados (Child B e C), sendo que 31,6 \% dos pacientes da amostra apresentaram perda em mais de um compartimento. Mudanças nos compartimentos corporais foram observadas, mesmo antes da detecção clínica de retenção hídrica. A avaliação nutricional multicompartimental, através da diluição isotópica, proporcionou uma avaliação mais precisa da DPC na cirrose, fornecendo dados que podem auxiliar no entendimento da sua fisiopatologia e no planejamento do suporte nutricional destes pacientes ${ }^{(12)}$.

\section{CONCLUSÃO}

Dos métodos apresentados, o modelo de avaliação multicompartimental de quatro compartimentos (massa celular corporal, água corporal total-intracelular e extracelular, gordura corporal, conteúdo mineral ósseo) proposto por FIGUEIREDO 
et al. ${ }^{(13)}$, se mostrou bastante sensível na detecção de desnutrição, mesmo em estágios iniciais da doença hepática e naqueles em que a desnutrição não havia sido detectada por outros métodos. O modelo multicompartimental proporcionou avaliação mais precisa da DPC na cirrose, fornecendo dados que auxiliarão no entendimento da sua fisiopatologia e no planejamento do suporte nutricional desses pacientes.

Mesmo com os bons resultados apresentados na avaliação multicompartimental, os métodos usados para sua detecção ainda esbarram em dificuldades técnicas e de alto custo.
Por outro lado, a FAM figura como método alternativo à avaliação multicompartimental pelo baixo custo, simplicidade e praticidade para aplicação em ambulatório. Além disso, mostrou-se eficaz para identificar os pacientes com maior risco de desenvolver complicações. Devido às complicações que a desnutrição pode causar a estes pacientes, é preferível se intervir com medidas nutricionais o mais precocemente possível, o que seria possível através dos dados fornecidos por este método de avaliação nutricional mais sensível, apesar de sua baixa especificidade.

Ritter L, Gazzola J. Nutritional evaluation of the cirrhotic patient: an objective, subjective or multicompartmental approach? Arq Gastroenterol. 2006;43(1):66-70.

Abstract - Background - Malnutrition due to liver disease is common and its assessment is difficult. The parameters of nutritional evaluation often used in clinical practice have limited use in cirrhotic patients. Many of the clinical signs of malnutrition are the result of the underlying hepatic disease which tends to confound and alter the nutritional diagnosis. Aim - To present a brief review of the different methods of nutritional evaluation together with their limitations and uses in cirrhotic patients. Conclusion - The multicompartmental approach for four compartments was shown to be considerably sensitive in the detection of malnutrition. However the ample use of this method is impaired due to technical difficulties and high costs. The handgrip strength appears to be a simple, cheap and effective alternative to detected malnutrition and risk of malnutrition in these patients. Is the most sensitive method and is able to predict a significant incidence of major complications in undernourished cirrhotic patients.

HEADINGS - Nutritional assessment. Liver cirrhosis. Hand strength.

\section{REFERÊNCIAS BIBLIOGRÁFICAS}

1. Álvares-da-Silva MR. Comparação entre a avaliação nutricional subjetiva global, índice prognóstico e força de aperto da mão não-dominante na avaliação nutricional do paciente cirrótico ambulatorial: a contribuição da dinamometria [dissertação]. Porto Alegre: Faculdade de Medicina da Universidade Federal do Rio Grande do Sul; 1995.

2. Álvares-da-Silva MR, Silveira TR. O estudo da força do aperto da mão não-dominante em indivíduos sadios. Determinação dos valores de referência para o uso da dinamometria. GED Gastroenterol Endosc Dig. 1998;17:203-6.

3. Álvares-da-Silva MR, Silveira TR. Comparison between handgrip strength, subjective global assessment, and prognostic nutritional index in assessing malnutrition and predicting clinical outcome in cirrhotic outpatients. Nutrition. 2005;21:113-7.

4. Andersen H, Borre M, Jakobsen J, Andersen PH, Vilstrup H. Decreased muscle strength in patients with alcoholic liver cirrhosis in relation to nutritional status, alcohol abstinence, liver function and neuropathy. Hepatology. 1998;27:1200-6.

5. Barbosa-Silva MCG, Barros AJD. Avaliação nutricional subjetiva: Parte 2 - Revisão de suas adaptações e utilizações nas diversas especialidades clínicas. Arq Gastroenterol. 2002;39:248-52.

6. Borges VC, Lima AO, D'Albuquerque LC, Waitzberg DL. Insuficiência hepática aguda e crônica. In: Waitzberg DL, editor. Nutrição oral, enteral e parenteral na prática clínica. 3a ed. Rio de Janeiro: Atheneu; 2000. p.1209-28.

7. Bramley PN, Oldroy B, Steward SP. How useful is skinfold anthropometry in liver cirrhosis? A comparison of techniques to measure body fat mass. Gut. 1992;33:526.

8. Brandão ABM, Silvério AO, Cassal AP, Pinheiro JB, Dittrich S, Bredemeier M. Etiologia da cirrose: experiência da Santa Casa de Misericórdia de Porto Alegre. Rev AMRIGS. 1995;39:25-31.

9. Chung RT, Podolsky DK. Cirrose e suas complicações. In: Harrison TR, editor Medicina interna. $15^{\mathrm{a}}$ ed. Rio de Janeiro: McGraw-Hill; 2002. p.1859.

10. Coppini LZ, Waitzberg DL, Ferrini MT, Teixeira-da-Silva ML, Gama-Rodrigues J, Ciosak SL. Comparação da avaliação nutricional subjetiva global x avaliação nutricional objetiva. Rev Assoc Med Bras. 1995;41:6-10.

11. Detsky SA, Baker, PJ, Mclaughlin RJ. What is subjective global assessment of nutritional states? J Parent Ent Nutr. 1987; 11:8-13

12. Figueiredo FA. Comparação entre dois modelos de avaliação do estado nutricional na cirrose hepática: tradicional e multicompartimental [dissertação]. São Paulo: Universidade Federal de São Paulo, Escola Paulista de Medicina; 2000.

13. Figueiredo FA, Dickson ER, Pasha TM, Porayko MK, Therneau TM, Malinchoc M, DiCecco SR, Francisco-Ziller NM, Kasparova P, Charlton MR. Utility of standard nutritional parameters in detecting body cell mass depletion in patients with end-stage liver disease. Liver Transpl. 2000;6:575-81.

14. Figueiredo FA, Perez RM, Kondo M. Effect of liver cirrhosis on body composition: evidence of significant depletion even in mild disease. J Gastroenterol Hepatol. 2005;20:209-16.
15. Fuller NJ, Jebb SA, Laskey MA, Coward WA, Elia M. Four-component model for the assessment of body composition in humans: comparison with alternative methods, and evaluation of density and hydration of fat-free mass. Clin Sci (Lond) 1992;82:687-93.

16. Gottschall CA, Álvares-da-Silva MR, Camargo AC, Burtett RM, Silveira TR. Avaliação nutricional de pacientes com cirrose pelo vírus da hepatite $\mathrm{C}$ : a aplicação da calorimetria indireta. Arq Gastroenterol. 2004;41:220-4.

17. Heymsfield SB, Waki M. Body composition in humans: advances in the development of multicompartment chemical models. Nutr Rev. 1991;49:97-108.

18. Jeejeebhoy KN, Detsky AS, Baker JP. Assessment of nutritional status. JPEN J Parenter Enteral Nutr. 1990;14(Suppl):193-6.

19. Lehnert ME, Clarke DD, Gibbons JG, Ward LC, Golding SM, Shepherd RW, Cornish $\mathrm{BH}$, Crawford DG. Estimation of body water compartments in cirrhosis by multiplefrequency bioelectrical-impedance analysis. Nutrition. 2001;17:31-4.

20. Leitão AVA, Castro CLN, Basile TM, Souza THS, Braulio VB. Avaliação da capacidade física e do estado nutricional em candidatos ao transplante hepático. Rev Assoc Med Bras. 2003;49:424-8.

21. Matos C, Porayko MK, Francisco-Ziller N, DiCecco S. Nutrition and chronic liver disease. J Clin Gastroenterol. 2002;35:391-7.

22. McCullough AJ. Malnutrition in liver disease. Liver Transpl. 2000;6(Suppl 1):85-96.

23. Mendenhall CL, Tosch T, Weesner RE, Garcia-Pont P, Goldberg SJ, Kiernan T, Seeff LB, Sorell M, Tamburro C, Zetterman R, Chedid A, Chen T, Rabin L. VA Cooperative study on alcoholic hepatitis. II: Prognostic significance of protein-calorie malnutrition. Am J Clin Nutr. 1986;43:213-8.

24. Merli M, Riggio O, Dally L. Does malnutrition affect survival in cirrhosis? Hepatology. 1996;23:1041-6.

25. Nutritional status in cirrhosis. Italian multicentre cooperative project on nutrition in liver cirrhosis. J Hepatol. 1994;21:317-25.

26. Parolin MB, Zaina FE, Lopes RW. Terapia nutricional no transplante hepático. Arq Gastroenterol. 2002;39:114-22.

27. Roongpisunthipong C, Sobhonslidsuk A, Nantiruj K, Songchitsomboon S. Nutritional assessment in various stages of liver cirrhosis. Nutrition. 2001;17:761-5.

28. Venegas Tresierra LF, Holguin-Mar RA, Yóza Yoshidaira M, Mormontoy Laurel W, Jara Cubas T, Polo Ramirez C, Vega Caico I. Evaluación y terapia nutricional en pacientes cirróticos del hospital "Edgardo Rebagliati Martins". Rev Gastroenterol Peru. 2002;22(1):13-8. 